PROCEEDINGS OF THE

AMERICAN MATHEMATICAL SOCIETY

Volume 139, Number 10, October 2011, Pages 3561-3571

S 0002-9939(2011)10761-5

Article electronically published on March 29, 2011

\title{
STABILITY OF SEMI-FREDHOLM PROPERTIES IN COMPLEX INTERPOLATION SPACES
}

\author{
KARL-HEINZ FÖRSTER AND KERSTIN GÜNTHER
}

(Communicated by Nigel J. Kalton)

\begin{abstract}
In this paper, we show that for interpolation morphisms $\vec{S}$ and the complex interpolation method the set of all $\theta \in(0,1)$ such that $S_{[\theta]}$ is a semi-Fredholm operator is open and the nullities, deficiencies and the indices of $S_{[\theta]}$ are locally constant on this set.
\end{abstract}

\section{INTRODUCTION}

Let $\vec{S}=\left(S_{0}, S_{1}\right)$ be an interpolation morphism between compatible couples of Banach spaces. E. Albrecht proved the following for the complex interpolation method (see [Alb84, Corollary 4.4]): If the operator $S_{[\lambda]}$ is semi-Fredholm for a $\lambda \in(0,1)$, then there exists $\delta>0$ such that for all $\theta \in(0,1)$ with $|\lambda-\theta|<\delta$, the operator $S_{[\theta]}$ is semi-Fredholm and ind $S_{[\theta]}=\operatorname{ind} S_{[\lambda]}$, nul $S_{[\theta]} \leq \operatorname{nul} S_{[\lambda]}$ and def $S_{[\theta]} \leq \operatorname{def} S_{[\lambda]}$. In this paper, we will prove that the nullities and the deficiencies (and the indices) of $S_{[\theta]}$ are actually constant in some neighborhood of $\lambda$.

There exist several papers on Fredholm properties of operators in various interpolation scales. For interpolation operators constructed with the real method, it is well-known that the set of parameters, for which these operators are semiFredholm, is open and the nullities, deficiencies and indices are locally constant on this set; see [Kra98, Corollary 4.6], [CS90, Theorem 3.1] and [AS, Theorem 3]. I.Ya. Shneiberg, see [Shn73] and [Shn74], was the first author who considered Fredholm properties of interpolation operators $A_{\alpha}$ between interpolation spaces $E_{\alpha}$ and $F_{\alpha}$, $\alpha \in[0,1]$. Under certain conditions on the families, the following was shown in Shn74, Lemma 7]: For $\alpha_{0} \in(0,1)$ such that $A_{\alpha_{0}}$ has positive minimum modulus, the operators $A_{\alpha}$ have positive minimum moduli and they have constant deficiencies in a neighborhood of $\alpha_{0}$. These results were extended to the interpolation of

Received by the editors February 27, 2009 and, in revised form, May 27, 2010 and August 25, 2010 .

2000 Mathematics Subject Classification. Primary 46B70, 47A53, 47A55.

Key words and phrases. Interpolation theory, complex interpolation method, duality, Fredholm operators, Punctured Neighborhood Theorem.

The authors thank the referee for helpful suggestions which improved the presentation of the paper.

(C)2011 American Mathematical Society

Reverts to public domain 28 years from publication 
families of Banach spaces; see CS91. N. Kalton and M. Mitrea considered interpolating families of operators $T_{w}$ between interpolation scales of quasi-Banach spaces $X_{w}$ and $Y_{w}$; see [KaMi98, Section 2]. One of their results is the following (see KaMi98, Theorem 2.3, Lemma 2.8]): For an element $w_{0}$ of the parameter space such that $T_{w_{0}}$ is surjective, the operators $T_{w}$ are surjective and they have constant nullities in a neighborhood of $w_{0}$.

E. Albrecht proved the results mentioned at the beginning of this introduction by lifting the operator $S_{[\theta]}$ to a graph (i.e. relation) and applying perturbation methods for relations. In [CS90, Corollary 2.6], this result was proved in the Fredholm case by different methods. N. Krugljak and M. Milman proved in KrMi04, Sections 6 and 7] injectivity and surjectivity results for the orbital methods using corresponding results for quotient spaces.

In the proof of our results, we will use a lifting procedure different from the one described in [Alb84, Section 4]. In the first step, we consider the case of finite deficiencies. For an interpolation couple $\vec{E}$, let $\mathcal{F}(\vec{E})$ denote the well-known class of analytic functions in the strip introduced by A.P. Calderón BL76, Chapter 4]). Each interpolation morphism $\vec{S}$ between two Banach couples $\vec{E}$ and $\vec{F}$ induces an operator $S_{\mathcal{F}}: \mathcal{F}(\vec{E}) \longrightarrow \mathcal{F}(\vec{F})$ and an operator $S_{[\lambda]}: E_{[\lambda]} \longrightarrow F_{[\lambda]}$ for $\lambda \in(0,1)$, where the interpolation spaces $E_{[\lambda]}$ and $F_{[\lambda]}$ are constructed with the complex method. Let $M_{\mathcal{F}(\vec{F})}$ denote the (closed, unbounded) operator of multiplication with the independent variable in $\mathcal{F}(\vec{F})$. For $S_{[\lambda]}$, we consider the operator $T_{\mathcal{F}, \lambda}:=\operatorname{row}\left(S_{\mathcal{F}}, \lambda \operatorname{id}_{\mathcal{F}(\vec{F})}-M_{\mathcal{F}(\vec{F})}\right)$ with domain $\mathcal{F}(\vec{E}) \times \mathrm{D}\left(M_{\mathcal{F}(\vec{F})}\right)$ in $\mathcal{F}(\vec{E}) \times \mathcal{F}(\vec{F})$ and range in $\mathcal{F}(\vec{F})$. From the results in Section 4 , it follows that $T_{\mathcal{F}, \lambda}$ is semiFredholm with finite deficiency if and only if $S_{[\lambda]}$ is semi-Fredholm with finite deficiency and the deficiencies coincide. Our main results now follow from the famous Punctured Neighborhood Theorem of T. Kato; see [Kat66, Theorem IV-5.31]. In the second step, the result for the finite nullity is proved by duality; here we use the characterization of dual spaces of interpolation spaces constructed with the complex method presented in [KPS82, Lemma IV-1.7].

The paper is organized as follows: In Section 2, we introduce the spaces $\mathcal{F}(\vec{E})$ and $\mathcal{K}\left(\vec{E}^{\prime}\right)$ for a compatible couple $\vec{E}$; these are spaces of analytic functions on the strip. In Section 3, we introduce operators induced by $\vec{S}$ and multiplication operators in $\mathcal{F}(\vec{E})$ and $\mathcal{K}\left(\vec{E}^{\prime}\right)$. In Section 4, we describe an abstract lifting procedure, which will be applied in Section 5 to obtain our main results; we show that a nonjumping version of the punctured neighborhood theorem holds.

We will use the notation and definitions of Kat66] for all relevant concepts for linear operators between Banach spaces. For the convenience of the reader, we recall the definition of semi-Fredholm operators (cf. [Kat66, IV-5.1]): A closed linear operator $T$ between Banach spaces $X$ and $Y$ is said to be semi-Fredholm if its range is closed and at least one of the spaces $\mathrm{N}(T)$ or the quotient $Y / \mathrm{R}(T)$ has finite dimension; here $\mathrm{N}(T)$ and $\mathrm{R}(T)$ denote the kernel and the range of $T$, respectively. In the following, nul $T$ and $\operatorname{def} T$ denote the dimension of the kernel of $T$ and $Y / \mathrm{R}(T)$, respectively. 


\section{Complex interpolation methods}

For a compatible couple $\vec{E}:=\left(E_{0}, E_{1}\right)$ (i.e. the Banach spaces $E_{0}$ and $E_{1}$ can be continuously embedded into a Hausdorff topological vector space), let $E_{\Delta}:=$ $\left(E_{0}, E_{1}\right)_{\Delta}:=E_{0} \cap E_{1}$ and $E_{\Sigma}:=\left(E_{0}, E_{1}\right)_{\Sigma}:=E_{0}+E_{1}$ be provided with the norms

$$
\begin{aligned}
& \left\|x_{\Delta}\right\|_{E_{\Delta}}:=\max \left\{\left\|x_{\Delta}\right\|_{E_{0}},\left\|x_{\Delta}\right\|_{E_{1}}\right\}, \\
& \left\|x_{\Sigma}\right\|_{E_{\Sigma}}:=\inf _{\substack{x_{\Sigma}=x_{0}+x_{1} \\
x_{0} \in E_{0}, x_{1} \in E_{1}}}\left\{\left\|x_{0}\right\|_{E_{0}}+\left\|x_{1}\right\|_{E_{1}}\right\} .
\end{aligned}
$$

It is well-known that $E_{\Delta}$ and $E_{\Sigma}$ are Banach spaces.

Now, we recall the definition of the complex interpolation method (see BL76, Chapter 4]). Let $\mathbb{S}_{0}:=\{z \in \mathbb{C}: \operatorname{Re} z \in(0,1)\}$. Its closure is denoted by $\mathbb{S}$. The space $\mathcal{F}(\vec{E})$ is the set of all bounded and continuous functions $f: \mathbb{S} \longrightarrow E_{\Sigma}$ such that $f$ is analytic on $\mathbb{S}_{0}, f(j+i t) \in E_{j}$ for all $t \in \mathbb{R}$, the map $t \mapsto f(j+i t)$ is continuous with respect to the norm on $E_{j}$ and $\|f(j+i t)\|_{E_{j}} \longrightarrow 0$ for $|t| \longrightarrow \infty$, where $j \in\{0,1\}$. If we provide $\mathcal{F}(\vec{E})$ with the norm

$$
\|f\|_{\mathcal{F}(\vec{E})}:=\max \left\{\sup _{t \in \mathbb{R}}\|f(i t)\|_{E_{0}}, \sup _{t \in \mathbb{R}}\|f(1+i t)\|_{E_{1}}\right\}
$$

then $\mathcal{F}(\vec{E})$ is a Banach space.

For $\lambda \in \mathbb{S}_{0}$, let $\left(E_{[\lambda]},\|\cdot\|_{[\lambda]}\right)$ denote the Banach space constructed with the complex interpolation method, i.e.

$$
\begin{aligned}
& E_{[\lambda]}:=\left\{x \in E_{\Sigma}: \exists f \in \mathcal{F}(\vec{E}) \text { with } f(\lambda)=x\right\}, \\
& \|x\|_{[\lambda]}:=\inf _{f \in \mathcal{F}(\vec{E})}\left\{\|f\|_{\mathcal{F}(\vec{E})}: f(\lambda)=x\right\} .
\end{aligned}
$$

The space

$$
\mathcal{N}_{\mathcal{F}(\vec{E}), \lambda}:=\{f \in \mathcal{F}(\vec{E}): f(\lambda)=0\}
$$

is closed in $\mathcal{F}(\vec{E})$. The quotient space $\mathcal{F}(\vec{E}) / \mathcal{N}_{\mathcal{F}(\vec{E}), \lambda}$ is isometrically isomorphic to $E_{[\lambda]}$.

Let $\vec{F}$ be a second compatible couple. Assume $S_{0}: E_{0} \longrightarrow F_{0}$ and $S_{1}: E_{1} \longrightarrow F_{1}$ are everywhere defined, linear and bounded operators. If $S_{0} x=S_{1} x$ for all $x \in E_{\Delta}$ (where the values are considered in $F_{\Sigma}$ ), then the pair $\vec{S}:=\left(S_{0}, S_{1}\right)$ is said to be an interpolation morphism between $\vec{E}$ and $\vec{F}$. As usual, its norm is

$$
\|\vec{S}\|_{\mathrm{Mor}}=\left\|\left(S_{0}, S_{1}\right)\right\|_{\mathrm{Mor}}:=\max \left\{\left\|S_{0}\right\|,\left\|S_{1}\right\|\right\} .
$$

For an interpolation morphism $\vec{S}$ between $\vec{E}$ and $\vec{F}$, the operators $S_{\Sigma}:=\left(S_{0}, S_{1}\right)_{\Sigma}$ : $E_{\Sigma} \longrightarrow F_{\Sigma}, S_{\Delta}:=\left(S_{0}, S_{1}\right)_{\Delta}: E_{\Delta} \longrightarrow F_{\Delta}$ and $S_{[\lambda]}: E_{[\lambda]} \longrightarrow F_{[\lambda]}$ are defined by

$$
\begin{aligned}
& S_{\Sigma}\left(x_{0}+x_{1}\right):=\left(S_{0}, S_{1}\right)_{\Sigma}\left(x_{0}+x_{1}\right):=S_{0} x_{0}+S_{1} x_{1}, \quad x_{0} \in E_{0}, x_{1} \in E_{1}, \\
& S_{\Delta} x_{\Delta}:=\left(S_{0}, S_{1}\right)_{\Delta} x_{\Delta}:=S_{\Sigma} x_{\Delta}=S_{0} x_{\Delta}=S_{1} x_{\Delta}, \quad x_{\Delta} \in E_{\Delta}, \\
& S_{[\lambda]} x:=S_{\Sigma} x, \quad x \in E_{[\lambda]} .
\end{aligned}
$$

All these operators are everywhere defined, linear and bounded with $\left\|S_{\Delta}\right\|,\left\|S_{\Sigma}\right\| \leq$ $\|\vec{S}\|_{\text {Mor }}$ and $\left\|S_{[\lambda]}\right\| \leq\left\|S_{0}\right\|^{1-\lambda}\left\|S_{1}\right\|^{\lambda}$.

The following observation plays an important role in the proof of our main result. For $\beta \in \mathbb{R}$, the operator in $\mathcal{F}(\vec{E})$ defined by $f \mapsto f(\cdot-i \beta)$ is an isometric isomorphism. Thus $E_{[\lambda]}=E_{[\lambda+i \beta]}$ with equal norms and $S_{[\lambda]}=S_{[\lambda+i \beta]}$ for all $\beta \in \mathbb{R}$ and $\lambda \in \mathbb{S}_{0}$. 
Now, we introduce the space described implicitly in [KPS82, Lemma IV-1.7] to characterize the dual space of an interpolation space constructed with the complex interpolation method.

Note that if $\vec{E}$ is a compatible couple such that $E_{\Delta}$ is dense in both $E_{0}$ and $E_{1}$, then $\vec{E}^{\prime}:=\left(E_{0}{ }^{\prime}, E_{1}{ }^{\prime}\right)$ is a compatible couple and it follows that $\left(E_{0}{ }^{\prime}, E_{1}{ }^{\prime}\right)_{\Delta}=E_{\Sigma}{ }^{\prime}$ and $\left(E_{0}{ }^{\prime}, E_{1}{ }^{\prime}\right)_{\Sigma}=E_{\Delta}{ }^{\prime}$ with equal norms; see [BL76, Theorem 2.7.1]. In this case, $\vec{E}$ is said to be a regular couple.

Definition 2.1. Let $\vec{E}$ be a regular couple. We define $\mathcal{K}\left(\vec{E}^{\prime}\right)$ as the set of all triples $\left(\psi, \psi_{0}, \psi_{1}\right)$ such that the following properties are fulfilled:

- $\psi: \mathbb{S}_{0} \longrightarrow\left(E_{0}{ }^{\prime}, E_{1}{ }^{\prime}\right)_{\Sigma}$ is analytic and bounded,

- $\psi_{j}: \mathbb{R} \longrightarrow E_{j}{ }^{\prime}$ is bounded for $j \in\{0,1\}$,

- for all $x \in E_{\Delta}$ and $j \in\{0,1\}$, we have for almost all $t \in \mathbb{R}$ that $\left\langle x, \psi_{j}(t)\right\rangle$ is the nontangential limit (see GM05]) of $\langle x, \psi(z)\rangle$ for $z \longrightarrow j+i t$.

The norm on the space $\mathcal{K}\left(\overrightarrow{E^{\prime}}\right)$ is

$$
\left\|\left(\psi, \psi_{0}, \psi_{1}\right)\right\|_{\mathcal{K}\left(\vec{E}^{\prime}\right)}:=\max \left\{\sup _{t \in \mathbb{R}}\left\|\psi_{0}(t)\right\|_{E_{0^{\prime}}}, \sup _{t \in \mathbb{R}}\left\|\psi_{1}(t)\right\|_{E_{1^{\prime}}}\right\} .
$$

The authors would like to thank J.B. Garnett and S. Kaijser for valuable hints concerning the following statements. They suggested proving these statements by using the conformal map from the strip to the upper half plane, changing variables and using the corresponding results for the half plane (see GM05]).

Let $\vec{E}$ be a regular couple and $\left(\psi, \psi_{0}, \psi_{1}\right) \in \mathcal{K}\left(\vec{E}^{\prime}\right)$.

- $\left\langle x, \psi_{j}(\cdot)\right\rangle$ is almost everywhere equal to a measurable function for $j \in\{0,1\}$ and all $x \in E_{\Delta}$.

- The Poisson formula holds for the strip $\mathbb{S}_{0}$; i.e. it is possible to obtain $\langle x, \psi(\cdot)\rangle$ from the boundary functions $\left\langle x, \psi_{0}(\cdot)\right\rangle$ and $\left\langle x, \psi_{1}(\cdot)\right\rangle$ via the Poisson kernels for all $x \in E_{\Delta}$.

- It follows for all $z \in \mathbb{S}_{0}$ that

$$
|\langle x, \psi(z)\rangle| \leq \max \left\{\sup _{t \in \mathbb{R}}\left|\left\langle x, \psi_{0}(t)\right\rangle\right|, \sup _{t \in \mathbb{R}}\left|\left\langle x, \psi_{1}(t)\right\rangle\right|\right\} .
$$

From the last inequality, we obtain

$$
\|\psi(z)\|_{\left(E_{0^{\prime}}{ }^{\prime}, E_{1}{ }^{\prime}\right)_{\Sigma}} \leq \max \left\{\sup _{t \in \mathbb{R}}\left\|\psi_{0}(t)\right\|_{E_{0^{\prime}}} \sup _{t \in \mathbb{R}}\left\|\psi_{1}(t)\right\|_{E_{1^{\prime}}}\right\} .
$$

Let the space $H^{\infty}\left(\mathbb{S}_{0},\left(E_{0}{ }^{\prime}, E_{1}{ }^{\prime}\right)_{\Sigma}\right)$ be the set of all bounded and analytic functions $\psi: \mathbb{S}_{0} \longrightarrow\left(E_{0}{ }^{\prime}, E_{1}{ }^{\prime}\right)_{\Sigma}$ provided with the norm

$$
\|\psi\|_{H^{\infty}\left(\mathbb{S}_{0},\left(E_{0}{ }^{\prime}, E_{1}{ }^{\prime}\right)_{\Sigma}\right)}:=\sup _{z \in \mathbb{S}_{0}}\|\psi(z)\|_{\left(E_{0}{ }^{\prime}, E_{1}{ }^{\prime}\right)_{\Sigma}}
$$

and, for $j \in\{0,1\}$, let the space $L^{\infty}\left(\mathbb{R}, E_{j}{ }^{\prime}\right)$ be the set of all measurable and essentially bounded functions $\psi: \mathbb{R} \longrightarrow E_{j}{ }^{\prime}$ provided with the norm

$$
\|\psi\|_{L^{\infty}\left(\mathbb{R}, E_{j^{\prime}}\right)}:=\underset{t \in \mathbb{R}}{\operatorname{ess} \sup }\|\psi(t)\|_{E_{j^{\prime}}} .
$$

Since $\left(\mathcal{K}\left(\overrightarrow{E^{\prime}}\right),\|\cdot\|_{\mathcal{K}\left(\vec{E}^{\prime}\right)}\right)$ is closed in $H^{\infty}\left(\mathbb{S}_{0},\left(E_{0}{ }^{\prime}, E_{1}{ }^{\prime}\right)_{\Sigma}\right) \times L^{\infty}\left(\mathbb{R}, E_{0}{ }^{\prime}\right) \times L^{\infty}\left(\mathbb{R}, E_{1}{ }^{\prime}\right)$, the space $\left(\mathcal{K}\left(\vec{E}^{\prime}\right),\|\cdot\|_{\mathcal{K}\left(\vec{E}^{\prime}\right)}\right)$ is a Banach space. It follows that $\mathcal{F}\left(\vec{E}^{\prime}\right) \subseteq \mathcal{K}\left(\vec{E}^{\prime}\right)$. 
For convenience, we sometimes consider $\left(\psi, \psi_{0}, \psi_{1}\right) \in \mathcal{K}\left(E^{\prime}\right)$ as a map from $\mathbb{S}$ into $\left(E_{0}{ }^{\prime}, E_{1}{ }^{\prime}\right)_{\Sigma}$; i.e.

$$
\left(\psi, \psi_{0}, \psi_{1}\right)(z):=\left\{\begin{array}{ll}
\psi(z) & \text { for } z \in \mathbb{S}_{0}, \\
\psi_{0}(t) & \text { for } z=i t, \\
\psi_{1}(t) & \text { for } z=1+i t,
\end{array} \quad z \in \mathbb{S} .\right.
$$

Let $\lambda \in \mathbb{S}_{0}$. Set

$$
\mathcal{N}_{\mathcal{K}\left(\vec{E}^{\prime}\right), \lambda}:=\left\{\left(\psi, \psi_{0}, \psi_{1}\right) \in \mathcal{K}\left(E^{\prime}\right): \psi(\lambda)=0\right\} .
$$

Since $\|\psi(\lambda)\|_{\left(E_{0}{ }^{\prime}, E_{1}{ }^{\prime}\right)_{\Sigma}} \leq\left\|\left(\psi, \psi_{0}, \psi_{1}\right)\right\|_{\mathcal{K}\left(\vec{E}^{\prime}\right)}$ by (1), we conclude that $\mathcal{N}_{\mathcal{K}\left(\vec{E}^{\prime}\right), \lambda}$ is closed in $\mathcal{K}\left(\vec{E}^{\prime}\right)$.

In [KPS82, Lemma IV-1.7 and Theorem IV-1.6], it is proved that the spaces $\mathcal{K}\left(\vec{E}^{\prime}\right) / \mathcal{N}_{\mathcal{K}\left(\vec{E}^{\prime}\right), \lambda}$ and $E_{[\lambda]}{ }^{\prime}$ are isometrically isomorphic. Again, we observe that the spaces $\mathcal{K}\left(\vec{E}^{\prime}\right) / \mathcal{N}_{\mathcal{K}\left(\vec{E}^{\prime}\right), \lambda}$ and $\mathcal{K}\left(\vec{E}^{\prime}\right) / \mathcal{N}_{\mathcal{K}\left(\vec{E}^{\prime}\right), \lambda+i \beta}$ are isometrically isomorphic for all $\beta \in \mathbb{R}$ and $\lambda \in \mathbb{S}_{0}$.

\section{MUltiplication OPERATORS AND INDUCED OPERATORS IN $\mathcal{F}(\vec{E})$ AND $\mathcal{K}\left(\vec{E}^{\prime}\right)$}

The operators introduced in this section will be essential for the proof of the main result in Section 5

Let $\vec{E}$ be a compatible couple. We define in $\mathcal{F}(\vec{E})$ the operator $M_{\mathcal{F}(\vec{E})}$ as the operator of multiplication by the independent variable, namely,

$$
\begin{aligned}
& \mathrm{D}\left(M_{\mathcal{F}(\vec{E})}\right):=\{f \in \mathcal{F}(\vec{E}):\{z \mapsto z f(z)\} \in \mathcal{F}(\vec{E})\}, \\
& \left(M_{\mathcal{F}(\vec{E})} f\right)(z):=z f(z) \quad \text { for all } z \in \mathbb{S} .
\end{aligned}
$$

The operator $M_{\mathcal{F}(\vec{E})}$ is linear and closed but not everywhere defined; indeed, let $0 \neq$ $x \in E_{\Delta}, q \in \mathbb{C} \backslash \mathbb{S}$ and $g(z):=\frac{x}{z-q}$ for all $z \in \mathbb{S}$. Then $g \in \mathcal{F}(\vec{E})$ but $g \notin \mathrm{D}\left(M_{\mathcal{F}(\vec{E})}\right)$. This operator was used in the proof of AM00, Theorem 4] to show that the local uniqueness-of-resolvent condition is always fulfilled. In [Gün08, Theorem 4.4], it is shown that this can be proved using the operator $\lambda \mathrm{id}_{\mathcal{F}(\vec{E})}-M_{\mathcal{F}(\vec{E})}$ and results of the perturbation theory stated in [För66, p. 58].

Proposition 3.1. Let $\vec{E}$ be a compatible couple and $\lambda \in \mathbb{S}_{0}$. The operator $\lambda \mathrm{id}_{\mathcal{F}(\vec{E})}-$ $M_{\mathcal{F}(\vec{E})}$ is linear, injective, closed and the range $\mathrm{R}\left(\lambda \mathrm{id}_{\mathcal{F}(\vec{E})}-M_{\mathcal{F}(\vec{E})}\right)$ is equal to the closed space $\mathcal{N}_{\mathcal{F}(\vec{E}), \lambda}$.

Proof. We only prove that $\mathrm{R}\left(\lambda \mathrm{id}_{\mathcal{F}(\vec{E})}-M_{\mathcal{F}(\vec{E})}\right) \supseteq \mathcal{N}_{\mathcal{F}(\vec{E}), \lambda}$; the other assertions are clear. Let $g \in \mathcal{N}_{\mathcal{F}(\vec{E}), \lambda}$. Since $\lambda \in \mathbb{S}_{0}$, there exists a function $f: \mathbb{S} \longrightarrow F_{\Sigma}$ such that $g(z)=(\lambda-z) f(z)$ for $z \in \mathbb{S}$. From $\lambda \in \mathbb{S}_{0}$ and $g \in \mathcal{F}(\vec{E})$, it follows easily that $f \in \mathrm{D}\left(\lambda \mathrm{id}_{\mathcal{F}(\vec{E})}-M_{\mathcal{F}(\vec{E})}\right)$ and $g=\left(\lambda \operatorname{id}_{\mathcal{F}(\vec{E})}-M_{\mathcal{F}(\vec{E})}\right) f \in \mathrm{R}\left(\lambda \mathrm{id}_{\mathcal{F}(\vec{E})}-M_{\mathcal{F}(\vec{E})}\right)$.

Let $\vec{S}$ be an interpolation morphism between the compatible couples $\vec{E}$ and $\vec{F}$. The linear operator $S_{\mathcal{F}}: \mathcal{F}(\vec{E}) \longrightarrow \mathcal{F}(\vec{F})$, defined by

$$
\left(S_{\mathcal{F}} f\right)(z):=S_{\Sigma}(f(z)) \quad \text { for all } z \in \mathbb{S},
$$


is everywhere defined and bounded with $\left\|S_{\mathcal{F}}\right\| \leq\|\vec{S}\|_{\text {Mor. }}$. It follows that $S_{\mathcal{F}}\left\{\mathcal{N}_{\mathcal{F}(\vec{E}), \lambda}\right\} \subseteq \mathcal{N}_{\mathcal{F}(\vec{F}), \lambda}$ for $\lambda \in \mathbb{S}_{0}$. We call $S_{\mathcal{F}}$ the operator induced by $\vec{S}$. If $S_{\Sigma}$ is injective, then $S_{\mathcal{F}}$ is injective.

Let $\lambda \in \mathbb{S}_{0}$. The linear operator $S_{\mathcal{F}, \lambda}: \mathcal{F}(\vec{E}) / \mathcal{N}_{\mathcal{F}(\vec{E}), \lambda} \longrightarrow \mathcal{F}(\vec{F}) / \mathcal{N}_{\mathcal{F}(\vec{F}), \lambda}$, defined by

$$
S_{\mathcal{F}, \lambda}\left(f+\mathcal{N}_{\mathcal{F}(\vec{E}), \lambda}\right):=S_{\mathcal{F}} f+\mathcal{N}_{\mathcal{F}(\vec{F}), \lambda}
$$

is well defined and bounded with $\left\|S_{\mathcal{F}, \lambda}\right\| \leq\left\|S_{\mathcal{F}}\right\|$. If $S_{\Sigma}$ is injective, then $S_{\mathcal{F}, \lambda}$ is injective.

The fact that $\mathcal{F}(\vec{E}) / \mathcal{N}_{\mathcal{F}(\vec{E}), \lambda}$ and $E_{[\lambda]}$ (correspondingly $\mathcal{F}(\vec{F}) / \mathcal{N}_{\mathcal{F}(\vec{F}), \lambda}$ and $F_{[\lambda]}$ ) are isometrically isomorphic shows that

$$
\operatorname{def} S_{\mathcal{F}, \lambda}=\operatorname{def} S_{[\lambda]} .
$$

Similarly, we introduce a multiplication operator in $\mathcal{K}\left(\vec{E}^{\prime}\right)$. Let $\vec{E}$ be a regular couple. We define $M_{\mathcal{K}\left(\vec{E}^{\prime}\right)}: \mathcal{K}\left(\vec{E}^{\prime}\right) \supseteq \mathrm{D}\left(M_{\mathcal{K}\left(\vec{E}^{\prime}\right)}\right) \longrightarrow \mathcal{K}\left(\overrightarrow{E^{\prime}}\right)$ with

$$
\begin{aligned}
& \mathrm{D}\left(M_{\mathcal{K}\left(\vec{E}^{\prime}\right)}\right):=\left\{\left(\psi, \psi_{0}, \psi_{1}\right) \in \mathcal{K}\left(\vec{E}^{\prime}\right):\left\{z \mapsto z\left(\psi, \psi_{0}, \psi_{1}\right)(z)\right\} \in \mathcal{K}\left(\vec{E}^{\prime}\right)\right\}, \\
& M_{\mathcal{K}\left(\vec{E}^{\prime}\right)}\left(\psi, \psi_{0}, \psi_{1}\right)(z):=z\left(\psi, \psi_{0}, \psi_{1}\right)(z)=\left\{\begin{array}{ll}
z \psi(z) & \text { for } z \in \mathbb{S}_{0}, \\
i t \psi_{0}(t) & \text { for } z=i t, \\
(1+i t) \psi_{1}(t) & \text { for } z=1+i t,
\end{array} \quad z \in \mathbb{S} .\right.
\end{aligned}
$$

The operator $M_{\mathcal{K}\left(\vec{E}^{\prime}\right)}$ is linear and closed but not everywhere defined. Indeed, for $x^{\prime} \in\left(E_{0}{ }^{\prime}, E_{1}{ }^{\prime}\right)_{\Delta}$, the triple $\left(\psi, \psi_{0}, \psi_{1}\right)$ defined by $\left(\psi, \psi_{0}, \psi_{1}\right)(z):=x^{\prime}$ for all $z \in \mathbb{S}$ is an element of $\mathcal{K}\left(\vec{E}^{\prime}\right)$ but not an element of the domain of $M_{\mathcal{K}\left(\vec{E}^{\prime}\right)}$.

The proof of the next proposition is similar to the proof of Proposition 3.1.

Proposition 3.2. Let $\vec{E}$ be a regular couple and $\lambda \in \mathbb{S}_{0}$. The operator $\lambda \mathrm{id}_{\mathcal{K}\left(\vec{E}^{\prime}\right)}-$ $M_{\mathcal{K}\left(\vec{E}^{\prime}\right)}$ is linear, injective, closed and the range $\mathrm{R}\left(\lambda \mathrm{id}_{\mathcal{K}\left(\vec{E}^{\prime}\right)}-M_{\mathcal{K}\left(\vec{E}^{\prime}\right)}\right)$ is equal to the closed space $\mathcal{N}_{\mathcal{K}\left(\vec{E}^{\prime}\right), \lambda}$.

Let $\vec{S}$ be an interpolation morphism between the regular couples $\vec{E}$ and $\vec{F}$. Then $\left(S_{0}{ }^{\prime}, S_{1}{ }^{\prime}\right)$ is an interpolation morphism between the regular couples $\left(F_{0}{ }^{\prime}, F_{1}{ }^{\prime}\right)$ and $\left(E_{0}{ }^{\prime}, E_{1}{ }^{\prime}\right)$. Indeed, we have for $y^{\prime} \in F_{0}{ }^{\prime} \cap F_{1}{ }^{\prime}$ and $x \in E_{\Delta}$ that

$$
\left\langle x, S_{0}{ }^{\prime} y^{\prime}\right\rangle=\left\langle S_{0} x, y^{\prime}\right\rangle=\left\langle S_{1} x, y^{\prime}\right\rangle=\left\langle x, S_{1}{ }^{\prime} y^{\prime}\right\rangle .
$$

Since $E_{\Delta}$ is dense in $E_{\Sigma}$, we conclude that $S_{0}{ }^{\prime} y^{\prime}=S_{1}{ }^{\prime} y^{\prime}$. It follows that $\left(S_{0}{ }^{\prime}, S_{1}{ }^{\prime}\right)_{\Sigma}=$ $S_{\Delta}{ }^{\prime}$ and $\left(S_{0}{ }^{\prime}, S_{1}{ }^{\prime}\right)_{\Delta}=S_{\Sigma}{ }^{\prime}$.

The linear operator $S_{\mathcal{K}}^{\prime}: \mathcal{K}\left(\vec{F}^{\prime}\right) \longrightarrow \mathcal{K}\left(\vec{E}^{\prime}\right)$, defined by

$$
S_{\mathcal{K}}^{\prime}\left(\psi, \psi_{0}, \psi_{1}\right):=\left(\left(S_{0}{ }^{\prime}, S_{1}{ }^{\prime}\right)_{\Sigma} \circ \psi, S_{0}{ }^{\prime} \circ \psi_{0}, S_{1}{ }^{\prime} \circ \psi_{1}\right),
$$

is everywhere defined and bounded with $\left\|S_{\mathcal{K}}^{\prime}\right\| \leq\left\|\left(S_{0}{ }^{\prime}, S_{1}{ }^{\prime}\right)\right\|_{\text {Mor }}$. It follows that $S_{\mathcal{K}}^{\prime}\left\{\mathcal{N}_{\mathcal{K}\left(\vec{F}^{\prime}\right), \lambda}\right\} \subseteq \mathcal{N}_{\mathcal{K}\left(\vec{E}^{\prime}\right), \lambda}$ for $\lambda \in \mathbb{S}_{0}$. We call $S_{\mathcal{K}}^{\prime}$ the operator induced by $\left(S_{0}{ }^{\prime}, S_{1}{ }^{\prime}\right)$. If $\left(S_{0}{ }^{\prime}, S_{1}{ }^{\prime}\right)_{\Sigma}$ is injective, then $S_{\mathcal{K}}^{\prime}$ is injective.

Let $\lambda \in \mathbb{S}_{0}$. We define $S_{\mathcal{K}, \lambda}^{\prime}: \mathcal{K}\left(\vec{F}^{\prime}\right) / \mathcal{N}_{\mathcal{K}\left(\vec{F}^{\prime}\right), \lambda} \longrightarrow \mathcal{K}\left(\vec{E}^{\prime}\right) / \mathcal{N}_{\mathcal{K}\left(\vec{E}^{\prime}\right), \lambda}$ by setting

$$
S_{\mathcal{K}, \lambda}^{\prime}\left(\left(\psi, \psi_{0}, \psi_{1}\right)+\mathcal{N}_{\mathcal{K}\left(\vec{F}^{\prime}\right), \lambda}\right):=S_{\mathcal{K}}^{\prime}\left(\psi, \psi_{0}, \psi_{1}\right)+\mathcal{N}_{\mathcal{K}\left(\overrightarrow{\left.E^{\prime}\right), \lambda}\right.} .
$$

This operator is everywhere defined, linear and bounded with $\left\|S_{\mathcal{K}, \lambda}^{\prime}\right\| \leq\left\|S_{\mathcal{K}}^{\prime}\right\|$. If $\left(S_{0}{ }^{\prime}, S_{1}{ }^{\prime}\right)_{\Sigma}$ is injective, then $S_{\mathcal{K}, \lambda}^{\prime}$ is injective. 
From the fact that $\mathcal{K}\left(\vec{E}^{\prime}\right) / \mathcal{N}_{\mathcal{K}\left(\vec{E}^{\prime}\right), \lambda}$ and $E_{[\lambda]^{\prime}}$ (correspondingly $\mathcal{K}\left(\vec{F}^{\prime}\right) / \mathcal{N}_{\mathcal{K}\left(\vec{F}^{\prime}\right), \lambda}$ and $\left.F_{[\lambda]}^{\prime}\right)$ are isometrically isomorphic, we conclude that

$$
\operatorname{def} S_{\mathcal{K}, \lambda}^{\prime}=\operatorname{def} S_{[\lambda]}^{\prime} .
$$

\section{ROW OPERATORS}

In this section, we state abstract results which we will apply in Section 5 to the operators of Section 3 .

Let $X, Y, Z$ be Banach spaces and $X_{0}$ and $Y_{0}$ be closed subspaces of $X$ and $Y$, respectively. Assume that $U: X \longrightarrow Y$ is everywhere defined, linear and bounded with $U\left\{X_{0}\right\} \subseteq Y_{0}$ and that $V: Z \supseteq \mathrm{D}(V) \longrightarrow Y$ is linear and closed with $\mathrm{R}(V)=Y_{0}$.

Let $U_{10}: X / X_{0} \longrightarrow Y / Y_{0}, U_{\mid 0}: X_{0} \longrightarrow Y_{0}$ and $V_{\mid 0}: Z \supseteq \mathrm{D}\left(V_{10}\right) \longrightarrow Y_{0}$ with $\mathrm{D}\left(V_{\mid 0}\right):=\mathrm{D}(V)$ be the operators induced by $U$ and $V$, respectively, to the corresponding spaces. The operators $i_{X_{0}}: X_{0} \longrightarrow X$ and $i_{Y_{0}}: Y_{0} \longrightarrow Y$ denote the embeddings corresponding to the inclusions $X_{0} \subseteq X$ and $Y_{0} \subseteq Y$, respectively. Then $V=i_{Y_{0}} V_{\mid 0}$. Let $q_{X_{0}}: X \longrightarrow X / X_{0}$ and $q_{Y_{0}}: Y \longrightarrow Y / Y_{0}$ be the canonical quotient maps. We have the following commutative diagram:

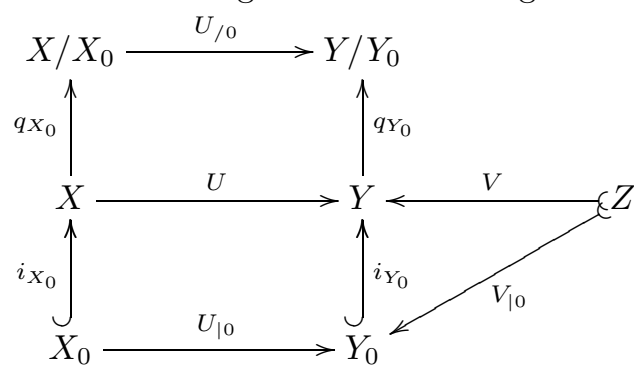

In the applications in Section [5, the spaces $X, Y=Z, X_{0}, Y_{0}$ and the operators $U, V, U_{/ 0}$ are considered to be as indicated in the following diagram:

\begin{tabular}{|c|c|c|c|c|c|c|}
\hline$X$ & $Y=Z$ & $X_{0}$ & $Y_{0}$ & $U$ & $V$ & $U_{/ 0}$ \\
\hline \hline $\mathcal{F}(\vec{E})$ & $\mathcal{F}(\vec{F})$ & $\mathcal{N}_{\mathcal{F}(\vec{E}), \theta}$ & $\mathcal{N}_{\mathcal{F}(\vec{F}), \theta}$ & $S_{\mathcal{F}}$ & $\theta \operatorname{id}_{\mathcal{F}(\vec{F})}-M_{\mathcal{F}(\vec{F})}$ & $S_{\mathcal{F}, \theta}$ \\
\hline $\mathcal{K}\left(\vec{F}^{\prime}\right)$ & $\mathcal{K}\left(\overrightarrow{E^{\prime}}\right)$ & $\mathcal{N}_{\mathcal{K}\left(\vec{F}^{\prime}\right), \theta}$ & $\mathcal{N}_{\mathcal{K}\left(\vec{E}^{\prime}\right), \theta}$ & $S_{\mathcal{K}}^{\prime}$ & $\theta \operatorname{id}_{\mathcal{K}\left(\vec{E}^{\prime}\right)}-M_{\mathcal{K}\left(\vec{E}^{\prime}\right)}$ & $S_{\mathcal{K}, \theta}^{\prime}$ \\
\hline
\end{tabular}

We are interested in the Fredholm properties of $U_{/ 0}$ and will connect them to the corresponding properties of $T:=\operatorname{row}(U, V): X \times Z \supseteq \mathrm{D}(T) \longrightarrow Y$ defined by

$$
\begin{gathered}
\mathrm{D}(T)=\mathrm{D}(\operatorname{row}(U, V)):=X \times \mathrm{D}(V), \\
T(x, z)=\operatorname{row}(U, V)(x, z):=U x+V z .
\end{gathered}
$$

Similarly, let $T_{\mid 0}: X_{0} \times Z \longrightarrow Y_{0}$ be the row operator row $\left(U_{\mid 0}, V_{\mid 0}\right)$. We denote by $\operatorname{proj}_{X \times Z, X}$ the projection from $X \times Z$ into $X$. Then

$$
q_{Y_{0}} T=q_{Y_{0}} U \operatorname{proj}_{X \times Z, X}=U_{/ 0} q_{X_{0}} \operatorname{proj}_{X \times Z, X}
$$

on $\mathrm{D}(T)$; i.e. the following diagram is commutative on $\mathrm{D}(T)$. (The operator $i_{X_{0} \times Z}$ denotes the embedding corresponding to the inclusion.) 


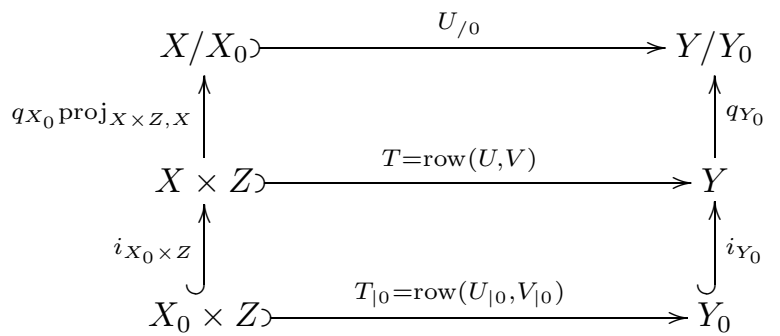

The following properties hold:

(a) $T$ is closed since $U$ is bounded and $V$ is closed,

(b) $q_{Y_{0}}\{\mathrm{R}(T)\}=\mathrm{R}\left(q_{Y_{0}} T\right)=\mathrm{R}\left(U_{/ 0} \operatorname{proj}_{X \times Z, X}\right)=\mathrm{R}\left(U_{/ 0}\right)$,

(c) $\mathrm{N}\left(q_{Y_{0}}\right)=Y_{0}=\mathrm{R}(V) \subseteq \mathrm{R}(T)$,

(d) $\mathrm{N}\left(q_{Y_{0}} T\right)=\mathrm{N}\left(\operatorname{row}\left(q_{Y_{0}} U, 0_{\mid \mathrm{D}(V)}\right)\right)=\mathrm{N}\left(q_{Y_{0}} U\right) \times \mathrm{D}(V)$,

(e) $\mathrm{N}\left(U_{/ 0}\right)=\left\{q_{X_{0}} x: x \in X\right.$ and $\left.0=U_{/ 0} q_{X_{0}} x=q_{Y_{0}} U x\right\}=q_{X_{0}}\left\{\mathrm{~N}\left(U_{/ 0} q_{X_{0}}\right)\right\}$ $=q_{X_{0}}\left\{\mathrm{~N}\left(q_{Y_{0}} U\right)\right\}=\mathrm{N}\left(q_{Y_{0}} U\right) / X_{0} \cong \mathrm{N}\left(q_{Y_{0}} T\right) /\left(X_{0} \times \mathrm{D}(V)\right)$

(f) $X_{0} \subseteq \operatorname{proj}_{X \times Z, X}\{\mathrm{~N}(T)\}$.

Assume that $V$ is injective. Then $V_{\mid 0}$ is injective and we have

(g) $\mathrm{N}(T)=\{(x, z) \in X \times \mathrm{D}(V): U x=-V z\}=\left\{\left(x,-V^{-1} U x\right): U x \in Y_{0}\right\}$,

(h) $\mathrm{N}\left(T_{\mid 0}\right)=\left\{\left(x_{0}, z\right) \in X_{0} \times \mathrm{D}\left(V_{\mid 0}\right): U_{\mid 0} x_{0}=-V_{\mid 0} z\right\}=\left\{\left(x_{0},-V_{\mid 0}^{-1} U_{\mid 0} x_{0}\right)\right.$ : $\left.x_{0} \in X_{0}\right\}$.

Proposition 4.1. Let $X, Y, Z$ be Banach spaces and $X_{0}, Y_{0}$ be closed subspaces of $X$ and $Y$, respectively. Assume that $U: X \longrightarrow Y$ is everywhere defined, linear and bounded with $U\left\{X_{0}\right\} \subseteq Y_{0}$ and $V: Z \supseteq \mathrm{D}(V) \longrightarrow Y$ is linear and closed with $\mathrm{R}(V)=Y_{0}$. Then for $T=\operatorname{row}(U, V)$, the following properties hold:

(i) The set $\mathrm{R}(T)$ is closed if and only if $\mathrm{R}\left(U_{/ 0}\right)$ is closed.

(ii) The spaces $Y / \mathrm{R}(T)$ and $\left(Y / Y_{0}\right) / \mathrm{R}\left(U_{/ 0}\right)$ are algebraically isomorphic. If $\mathrm{R}(T)$ (or equivalently $\mathrm{R}\left(U_{/ 0}\right)$ is closed; see (i)), then these spaces are isometrically isomorphic.

(iii) Assume $V$ is injective. Then the spaces $\mathrm{N}(T) / \mathrm{N}\left(T_{00}\right)$ and $\mathrm{N}\left(U_{/ 0}\right)$ are isomorphic with equivalent norms.

Proof. (i) Assume $\mathrm{R}(T)$ is closed. Since $\mathrm{R}\left(q_{Y_{0}}\right)$ is closed, we obtain that $q_{Y_{0}}\{\mathrm{R}(T)\}$ $=\mathrm{R}\left(U_{10}\right)$ is closed from (b), (c) above and [Kat58, Lemma 331].

Conversely, assume $\mathrm{R}\left(U_{/ 0}\right)$ is closed. Let $\left\{y_{n}\right\}_{n \in \mathbb{N}} \subseteq \mathrm{R}(T), y \in Y$ be such that $y_{n} \longrightarrow y$. Then $q_{Y_{0}} y_{n} \longrightarrow q_{Y_{0}} y$ and, by (b) above, it follows that $q_{Y_{0}} y_{n} \in$ $q_{Y_{0}}\{\mathrm{R}(T)\}=\mathrm{R}\left(U_{/ 0}\right)$. Since $\mathrm{R}\left(U_{/ 0}\right)$ is closed, we obtain $q_{Y_{0}} y \in \mathrm{R}\left(U_{/ 0}\right)$. Thus $y \in \mathrm{R}(T)+Y_{0}=Y_{0}$ by (b) and (c) above.

(ii) The operator $q_{Y_{0}}: Y \longrightarrow Y / Y_{0}$ is everywhere defined, linear, surjective and $q_{Y_{0}}\{\mathrm{R}(T)\}=\mathrm{R}\left(U_{/ 0}\right)$ by (b) above. Then it follows directly that its induced operator from $Y / \mathrm{R}(T)$ into $\left(Y / Y_{0}\right) / \mathrm{R}\left(U_{/ 0}\right)$ is everywhere defined, linear and surjective. The injectivity follows from (b) above.

(iii) We consider the operator from $\mathrm{N}(T)$ into $\mathrm{N}\left(U_{/ 0}\right)$ defined by

$$
(x, z) \mapsto q_{X_{0}} x, \quad(x, z) \in \mathrm{N}(T) .
$$

This operator is well defined and surjective with the kernel $\left\{\left(x,-V^{-1} U x\right): x \in\right.$ $\left.X_{0}\right\}=\mathrm{N}\left(T_{\mid 0}\right)$. Moreover, it is the restriction of the bounded operator $q_{X_{0}} \operatorname{proj}_{X \times Z, X}$ to the spaces $\mathrm{N}(T)$ and $\mathrm{N}\left(U_{/ 0}\right)$. 
In the following section, we will need the corollary below, itself a direct consequence of Proposition 4.1 .

Corollary 4.2. Under the assumptions of Proposition 4.1, we have the following equivalence:

$$
\operatorname{def} T=\operatorname{def} \operatorname{row}(U, V)=n<\infty \Longleftrightarrow \operatorname{def} U_{/ 0}=n<\infty .
$$

\section{MAin RESUlt AND COROLlARIES}

Our main result is the following 'Punctured Neighborhood Theorem' for the complex interpolation method. The proof of this theorem relies on the famous Punctured Neighborhood Theorem of T. Kato; see [Kat66, Theorem IV-5.31] (cf. Kat58, Theorem 3 and Theorem 5]). We use the concept of semi-Fredholm operators as in [Kat66, IV-5.1]; see also the end of Section 1.

Theorem 5.1. Let $\vec{S}$ be an interpolation morphism between the compatible couples $\vec{E}$ and $\vec{F}$.

(i) Assume $\lambda \in \mathbb{S}_{0}$ such that $\operatorname{def} S_{[\lambda]}<\infty$. Then there exists $\delta>0$ such that

$$
\operatorname{def} S_{[\lambda]}=\operatorname{def} S_{[\theta]}
$$

for all $\theta \in \mathbb{D}_{\lambda, \delta} \cap \mathbb{S}_{0}$, where $\mathbb{D}_{\lambda, \delta}:=\{z \in \mathbb{C}:|z-\lambda|<\delta\}$.

(ii) Assume $\vec{E}$ and $\vec{F}$ are regular and $\lambda \in \mathbb{S}_{0}$ such that $\operatorname{def} S_{[\lambda]}{ }^{\prime}<\infty$. Then there exists $\delta>0$ such that

$$
\operatorname{def} S_{[\lambda]}^{\prime}=\operatorname{def} S_{[\theta]}{ }^{\prime}
$$

for all $\theta \in \mathbb{D}_{\lambda, \delta} \cap \mathbb{S}_{0}$.

Proof. (i) For $\theta \in \mathbb{S}_{0}$, let $T_{\mathcal{F}, \theta}:=\operatorname{row}\left(S_{\mathcal{F}}, \theta \operatorname{id}_{\mathcal{F}(\vec{F})}-M_{\mathcal{F}(\vec{F})}\right)$ with domain $\mathcal{F}(\vec{E}) \times$ $\mathrm{D}\left(M_{\mathcal{F}(\vec{F})}\right)$ in $\mathcal{F}(\vec{E}) \times \mathcal{F}(\vec{F})$ and range in $\mathcal{F}(\vec{F})$. It follows that def $S_{[\lambda]}=\operatorname{def} S_{\mathcal{F}, \lambda}$; see (2) in Section 3. From Proposition 3.1 and Corollary 4.2 (cf. the scheme in Section (4), it follows that $T_{\mathcal{F}, \lambda}$ is semi-Fredholm and $\operatorname{def} T_{\mathcal{F}, \lambda}=\operatorname{def} S_{\mathcal{F}, \lambda}$ is finite. We have

$$
T_{\mathcal{F}, \theta}=(\theta-\lambda) \operatorname{row}\left(0, \operatorname{id}_{\mathcal{F}(\vec{F})}\right)+T_{\mathcal{F}, \lambda}
$$

for all $\theta \in \mathbb{S}_{0}$. Then we obtain from the well-known Punctured Neighborhood Theorem of T. Kato (see [Kat66, Theorem IV-5.31]) that there exist $\delta>0$ and a nonnegative integer $r$ such that $\operatorname{def}\left(T_{\mathcal{F}, \theta}\right)=\operatorname{def}\left(T_{\mathcal{F}, \lambda}\right)-r$ for all $\theta \in\left(\mathbb{D}_{\lambda, \delta} \backslash\{\lambda\}\right) \cap \mathbb{S}_{0}$. From Proposition 3.1 and Corollary 4.2 (cf. the scheme in Section 44), we conclude that $\operatorname{def}\left(S_{\mathcal{F}, \theta}\right)=\operatorname{def}\left(S_{\mathcal{F}, \lambda}\right)-r$ for all $\theta \in\left(\mathbb{D}_{\lambda, \delta} \backslash\{\lambda\}\right) \cap \mathbb{S}_{0}$. Thus $\operatorname{def}\left(S_{[\theta]}\right)=$ $\operatorname{def}\left(S_{[\lambda]}\right)-r$ for all $\theta \in\left(\mathbb{D}_{\lambda, \delta} \backslash\{\lambda\}\right) \cap \mathbb{S}_{0}$ by (2) in Section 3 . Since $F_{[\lambda]}=F_{\left[\lambda+i \frac{\delta}{2}\right]}$ and $S_{[\lambda]}=S_{\left[\lambda+i \frac{\delta}{2}\right]}$, it follows that $r=0$.

(ii) In the same way as (i), we prove (ii) with Proposition 3.2 and (3) in Section 3 considering the operator $\operatorname{row}\left(S_{\mathcal{K}}^{\prime}, \theta \operatorname{id}_{\mathcal{K}\left(\vec{E}^{\prime}\right)}-M_{\mathcal{K}\left(\overrightarrow{E^{\prime}}\right)}\right)$. 
From Theorem [5.1] and [Alb84, Corollary 4.4], we then obtain the following corollary.

Corollary 5.2. Let $\vec{S}$ be an interpolation morphism between the regular couples $\vec{E}$ and $\vec{F}$. Assume $\lambda \in \mathbb{S}_{0}$ such that $S_{[\lambda]}$ is semi-Fredholm. Then there exists $\delta>0$ such that $S_{[\theta]}$ is semi-Fredholm and

$$
\operatorname{nul} S_{[\lambda]}=\operatorname{nul} S_{[\theta]}, \quad \operatorname{def} S_{[\lambda]}=\operatorname{def} S_{[\theta]}, \quad \text { ind } S_{[\lambda]}=\operatorname{ind} S_{[\theta]}
$$

for all $\theta \in \mathbb{D}_{\lambda, \delta} \cap \mathbb{S}_{0}$.

The previous corollary improves upon results of E. Albrecht stated in Alb84, Corollary 4.4] concerning the weak inequalities of the nullities and deficiencies, in the sense that here we show that these inequalities are, in fact, equalities.

Corollary 5.3. Let $\vec{S}$ be an interpolation morphism between the regular couples $\vec{E}$ and $\vec{F}$. The set

$$
\left\{\theta \in \mathbb{S}_{0}: S_{[\theta]} \text { is semi-Fredholm }\right\}
$$

is open and the nullities, deficiencies and the indices of $S_{[\cdot]}$ are locally constant on this set.

\section{REFERENCES}

[Alb84] E. Albrecht, Spectral interpolation, Oper. Theory Adv. App. 14 (1984), 13-37. MR.789606 (86j:46071)

[AM00] E. Albrecht, V. Müller, Spectrum of interpolated operators, Proc. Amer. Math. Soc. 129 (2000), no. 3, 807-814. MR.1804050 (2001j:47001)

[AS] E. Albrecht, K. Schindler, Spectra of operators on real interpolation spaces, preprint.

[BL76] J. Bergh, J. Löfström, Interpolation spaces, Springer, 1976. MR0482275 (58:2349)

[CS90] W. Cao, Y. Sagher, Stability of Fredholm properties on interpolation scales, Ark. Mat. 28 (1990), no. 2, 249-258. MR1084014 (92e:46146)

[CS91] W. Cao, Y. Sagher, Stability in interpolation of families of Banach spaces, Proc. Amer. Math. Soc. 112 (1991), no. 1, 91-100. MR1031449 (91h:46123)

[För66] K.-H. Förster, Über die Invarianz einiger Räume, die zum Operator $T-\lambda A$ gehören, Arch. Math. 17 (1966), 56-64. MR0203467 (34:3318)

[GM05] J.B. Garnett, D.E. Marshall, Harmonic measure, Cambridge Univ. Press, 2005. MR 2150803 (2006g:31002)

[Gün08] K. Günther, Unbounded linear operators on interpolation spaces, PhD thesis, Technische Universität Berlin, 2008.

[KaMi98] N. Kalton, M. Mitrea, Stability results on interpolation scales of quasi-Banach spaces and applications, Trans. Amer. Math. Soc. 350 (1998), no. 10, 3903-3922. MR1443193 (98m:46094)

[Kat58] T. Kato, Perturbation theory for nullity, deficiency and other quantities of linear operators, J. Anal. Math. 6 (1958), 261-322. MR0107819 (21:6541)

[Kat66] T. Kato, Perturbation theory for linear operators, Springer, 1966. MR 0203473 (34:3324)

[KPS82] S.G. Krein, J.I. Petunin, E.M. Semenov, Interpolation of linear operators, Amer. Math. Soc., 1982. MR649411 (84j:46103)

[Kra98] M. Krause, Fredholm theory of interpolation morphisms, Oper. Theory Adv. App. 103 (1998), 219-231. MR.1635025 (99h:46136)

[KrMi04] N. Krugljak, M. Milman, A distance between orbits that controls commutator estimates and invertibility of operators, Advances in Mathematics 182 (2004), 78-123. MR2028497 (2005d:46155) 
[Shn73] I.Ya. Shneiberg, On the solvability of linear equations in interpolational families of Banach spaces, Soviet Math. Dokl. 14 (1973), no. 5, 1328-1331. MR0636330 (58:30443)

[Shn74] I.Ya. Shneiberg, Spectral properties of linear operators in interpolation families of Banach spaces, Mat. Issled. 9 (1974), 214-229. MR0634681 (58:30362)

Technische Universität Berlin, MA 6-4, Strasse des 17. Juni 136, 10623 Berlin, GerMANY

E-mail address: foerster@math.tu-berlin.de

Technische Universität Berlin, MA 6-4, Strasse des 17. Juni 136, 10623 Berlin, GerMANY

E-mail address: guenther@math.tu-berlin.de 